Background Microvesicles (MVs) expressing the type 1 interferon (IFN)-inducible protein galectin-3 binding protein (G3BP) may play a pathogenic role in systemic lupus erythematosus (SLE). Co-expression of DNA on such MVs may render them immunogenic and target for anti-dsDNA antibodies. Little is known about the mechanisms underlying generation of this MV population. In this study, we investigated how Toll-like receptors, interferon- (IFN-) and $\mathrm{T}$ cells are related hereto in healthy subjects.

Methods Peripheral blood mononuclear cells (PBMCs) isolated from 12 healthy donors were stimulated in-vitro for 24 hours with a series of TLR-agonists or the T-cell activating antibody OKT3 or were subjected to apoptosis by incubation with staurosporine. MVs in the supernatants were subsequently isolated by differential centrifugation and were quantified and characterized with respect to expression of G3BP and DNA by flow cytometry.

Results Stimulation of PBMCs with the TLR9-agonist and strong IFN- inducer ODN2395 significantly increased the release of MVs expressing G3BP. A large proportion of these MVs expressed augmented levels of DNA on their surface. The production of MVs with this phenotype was markedly enhanced by co-stimulation of $\mathrm{T}$ cells. Furthermore, dependency on IFN- in the generation of G3BPexpressing MVs was indicated by a marked reduction following addition of the IFN- inhibitor IFN alpha-IFNAR-IN1 hydrochloride.

Conclusions The release of G3BP-expressing MVs from healthy donor PBMCs is induced by stimulation of TLR9 in an IFNdependent manner. The co-expression of DNA accessible for anti-DNA antibodies on these MVs may render them relevant in lupus pathogenesis.

Funding Source(s): Lundbeck Foundation, Rigshospitalets Fund for Research, Gigtforeningen

Effect of IFN- inhibition on TLR9-induced release of MVs from mononuclear cells.

\section{ZOSTER INFECTIONS INCREASE THE RISK OF FLARES IN PATIENTS WITH SYSTEMIC LUPUS ERYTHEMATOSUS}

${ }^{1}$ Fangfang Sun*, ${ }^{2}$ Shuang Ye. ${ }^{1}$ Ren Ji Hospital, South Campus, Shanghai Jiaotong University; '2Department of Rheumatology, South Campus, Ren Ji Hospital, School of Medicine, Shanghai Jiao Tong University

\subsection{6/lupus-2019-Ism.132}

Background The aim of this cohort study is to explore whether zoster infection could increase the risk of flares in patients with systemic lupus erythematosus.

Methods Patients who had zoster infection between January 2013 and April 2018 were included from the SLE database of Shanghai Ren Ji Hospital, South Campus. Patients with bacterial infections including urinary tract infection and bacterial pneumonia were selected as controls. Severe infections including fungi infections and septic shock were excluded. Besides, 200 SLE patients at the same time were set as general populations by systemic sampling. Baseline period was defined as three months before infections or admissions for general population, and risk period was within three months after infections or admissions. Characteristics and treatment strategies of baseline period including gender, age, disease duration, average daily dose of prednisone and immunosuppressive agents were collected. Flare was defined by SELENE SLEDAI criterion. Flare free

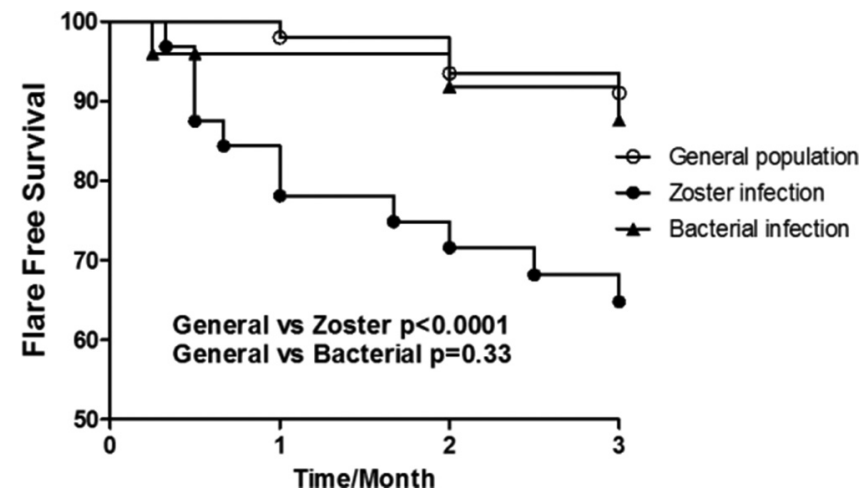

Abstract 132 Figure 1 Kaplan Meier curve showed that compared to general population, more patients with a history of zoster infectionwithin 3 months had a relapse of disease, while bacterial infections did not seem to affect the relapse rate

survival was assessed by Kaplan-Meier curve. Cox regression analysis was performed to identify risk factors of flares.

Results 33 with zoster infections, 18 with urinary tract infections and 7 patients with bacterial pneumonia were identified in this period. 13 relapses in 11 patients were observed, but there were only 3 relapses in bacterial infection group. Kaplan Meier curve showed that compared to general population, more patients with a recent history of zoster infection within 3 months had a flare $(33.3 \%$ vs $9 \%, \mathrm{p}=0.0034)$, while bacterial infections did not seem to affect the flare rate $(12 \%$ vs $9 \%, \mathrm{p}=0.4755)$. Furthermore, after adjusting important covariates, cox regression analysis confirmed that prior zoster infection within three months was a risk factor of flares in SLE patients $(\mathrm{p}<0.001$, HR 4.7).

Conclusions In patients with SLE, recent zoster infection within three months may be associated with increased risk of flares.

Funding Source(s): Kaplan Meier Curve of patients in three groups

\section{A YOUNG GIRL WITH LUPUS, RECURRENT PERICARDIAL EFFUSION AND CARDIAC TAMPONADE}

${ }^{1}$ Gummadi Anjani*, 'Rakesh Pilania, ${ }^{2}$ Anju Gupta, ${ }^{3}$ Deepti Suri, ${ }^{3}$ Surjit Singh. ${ }^{1}$ Dept. of Pediatrics, Allergy- Immunology Unit, Postgraduate Institute of Medical Education and Research; ${ }^{2}$ PGIMER Chandigarh; ${ }^{3}$ Postgraduate Institute of Medical Education and Research, Chandigarh, India

\subsection{6/lupus-2019--Ism.133}

Background Pleuritis and pericarditis,with or without effusion, are the commonest pulmonary and cardiovascular manifestations in children with systemic lupus erythematosus(SLE).However,SLE presenting as an isolated pericardial effusion with cardiac tamponade is distinctly unusual.

Methods We report one such case who went on to develop recurrent episodes of pericardial effusion and tamponade.

Results A-14-year-old girl presented with high grade fever, progressive dyspnea,pedal edema,periorbital puffiness for 7 days.She also had history of malar rash and photosensitivity. There was no history of oral ulcer and hair loss.Physical examination showed a prominent malar rash;heart rate 140/ min;respiratory-rate 40/min;muffled heart sounds; 


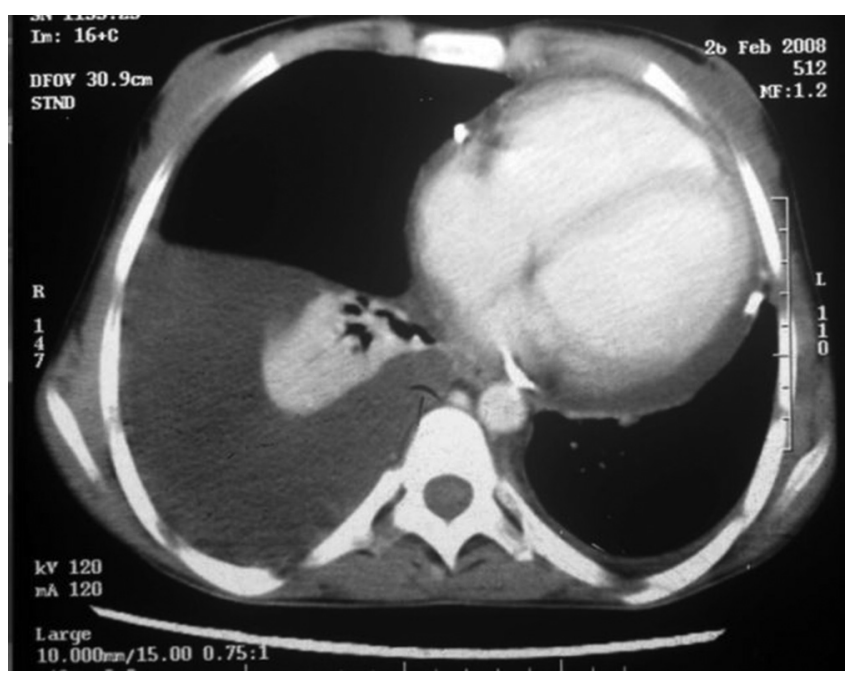

Abstract 133 Figure 1 Pericardial effusion and pleural effusion

hepatomegaly and ascites.She was initiated on decongestive measures with furosemide and digoxin.Chest X-ray showed cardiomegaly(cardiothoracic ratio 68\%);2D-echocardiography (2D-ECHO) revealed cardiomegaly,pericardial effusion and features consistent with cardiac tamponade.She underwent emergency pericardiocentesis, $400 \mathrm{ml}$ of serosanguineous fluid was drained followed by prompt clinical improvement.Investigations showed hemoglobin $90 \mathrm{~g} / \mathrm{L}$; total leukocyte count 1.3 $\times 106 / \mathrm{L}$ and lymphocyte count $0.18 \times 106 / \mathrm{L}$;platelets of $356 \times 109 /$ L.Urinalysis and renal function tests were unremarkable.Tuberculin skin test was non-reactive.Thyroid function tests were normal.In view of febrile illness, malar rash, photosensitivity, pericardial effusion,cardiac tamponade and lymphopenia,SLE was considered. Work-up showed positive antinuclear antibody (ANA)4+diffuse ANA positivity;positive anti-double stranded DNA(anti-dsDNA)-60(normal:4.2 IU/ $\mathrm{ml}$ );C3 $51.8 \mathrm{mg} \%$ (normal $50-150 \mathrm{mg} \%$ ).Skin biopsy revealed IgG and C3 deposits.Pericardial fluid analysis revealed low complement(C3<12 mg\%);ANA positivity and positive LE cells.Bacterial culture of pericardial fluid and polymerase chain reaction to Mycobacterium tuberculosis were negative.Pericardial fluid showed no malignant cells on cytological examination.A diagnosis of SLE was offered based on positive clinical and immunologic findings.Pulse intravenous methylprednisolone was given $(30 \mathrm{mg} / \mathrm{kg} /$ day for 5 days $)$ followed by oral prednisolone(starting at $2 \mathrm{mg} / \mathrm{kg} /$ day and tapered thereof).2D-ECHO showed a reduced volume of pericardial fluid with no additional reaccumulation and normal heart function. She remained well for the next 3 years on hydroxychloroquine. However,at age of 17 years she presented with respiratory distress,oral ulcerations, right pleural effusion and recurrence of pericardial effusion and cardiac tamponade.This required pigtail insertion for pericardiocentesis for 8 days.There was laboratory evidence for disease activity(antids-DNA index 2.24;normal <1.1).She responded to oral prednisolone $(2 \mathrm{mg} / \mathrm{kg} / \mathrm{day})$.A similar episode occurred a year later at 18 years with pericardial and pleural effusion and for this she was given intravenous methylprednisolone pulses for 5 days.In view of recurrent pericarditis, she has been initiated on colchicine $(1 \mathrm{mg} /$ day $)$ following which there has been no recurrence of symptoms.

Conclusions Pericardial effusion with cardiac tamponade at presentation of childhood lupus is distinctly unusual and so is the rarity of recurrent pericardial effusion with cardiac tamponade.Our patient continued to have recurrences on follow-up that responded to glucocorticoids.

Funding Source(s): None

\section{MORTALITY RISK PREDICTION IN LUPUS PATIENTS COMPLICATING WITH INVASIVE INFECTION IN THE EMERGENCY DEPARTMENT}

${ }^{1} Y_{i}$ Chen*, ${ }^{2}$ Wanlong Wu, ${ }^{2}$ Shuang Ye, ${ }^{3}$ Fangfang Sun, ${ }^{1} J u n$ Ma. ${ }^{1}$ Department of Emergency, South Campus, Ren Ji Hospital, School of Medicine, Shanghai Jiao Tong University; '2Department of Rheumatology, South Campus, Ren Ji Hospital, School of Medicine, Shanghai Jiao Tong University; ${ }^{3}$ Ren Ji Hospital, South Campus, Shanghai Jiaotong University

\subsection{6/lupus-2019-Ism.134}

Background Infection remains an important and leading cause of mortality in lupus. We aimed to establish a clinical prediction model for the 3 month all-cause mortality of patients with systemic lupus erythematosus (SLE) and invasive infection in the emergency department.

Methods SLE patients classified by 1997 ACR criteria complicating with invasive infection when admitted into the emergency department of our center between May 2015 and June 2018 were included. Invasive infection was defined as deep infection with definite evidence of pathogens or globally judged by the treating physician combining symptoms, lab and imaging tests. Patients clinical and laboratory characteristics at admission were retrospectively collected as baseline data. The outcome was all-cause death within 3 months since baseline.

Candidate predictors for multivariable logistic regression were selected by expert opinion based on clinical significance, previous studies and feasibility. A prediction model for allcause mortality was established by combining independent predictors and evaluated by Receiver Operating Characteristic (ROC) curve analysis.

Results A total of 50/130 (38.5\%) included patients died cumulatively within 3 month follow-up. Patients were predominantly female (91\%) with a mean disease duration of 6.6 years. One hundred and eight (83.1\%) patients had lung infection, while $23(17.7 \%)$ patients had blood stream infection.

In the final multivariable logistic regression model, lymphocyte count $<800 / \mathrm{ul}$, urea $>7.6 \mathrm{mmol} / \mathrm{L}$, maximum prednisone dose in the past60 mg/d, qSOFA score and age at admission were independently predictive for all-cause mortality. However, the history of hydroxychloroquine use was protective.

In a combined prediction model, the six predictors were weighted by OR values, making the LUPHAS score ranging from 5 to 22 (table). All patients could be categorized to three group: low-risk (score 5-10), medium-risk (score 11-16) and high-risk (score 17-22). The mortalities were $11.1 \%$ (4/ $36), 32.8 \%(22 / 67)$ and $100 \%(22 / 22)$ in low-risk, mediumrisk and high-risk patients, respectively. ROC curve analysis indicated that LUPHAS score could effectively predict all-cause mortality in this population $(\mathrm{AUC}=0.8595 \% \mathrm{CI}=0.78-0.92$ ). Furthermore, LUPHAS score performed better than the sole qSOFA score $(\mathrm{AUC}=0.6995 \% \mathrm{CI}=0.59-0.78)$ in our cohort. The discriminatory performance of LUPHAS score was also superior than CURB-65 score (AUC $=0.6995 \% \mathrm{CI}=0.59-0.80$ ) in the subgroup of patients with lung infection $(n=108)$.

Conclusions In this large emergency cohort of lupus patients complicating with invasive infection, an impressive high 